le portiQue $\begin{array}{ll}\text { Le Portique } \\ \text { Revue de philosophie et de sciences humaines }\end{array}$

33 | 2014

Straub !

\title{
Débat Sons et Images
}

Philippe Lafosse, Jacques Drillon, Renato Berta, Benoît Turquety et Léon Garcia Jordan

\section{OpenEdition}

\section{Journals}

Édition électronique

URL : http://journals.openedition.org/leportique/2771

DOI : $10.4000 /$ leportique.2771

ISSN : $1777-5280$

Éditeur

Association "Les Amis du Portique"

Édition imprimée

Date de publication : 1 mai 2014

ISSN : 1283-8594

\section{Référence électronique}

Philippe Lafosse, Jacques Drillon, Renato Berta, Benoît Turquety et Léon Garcia Jordan, « Débat Sons et Images ", Le Portique [En ligne], 33 | 2014, document 2, mis en ligne le 05 février 2016, consulté le 12 avril 2021. URL : http://journals.openedition.org/leportique/2771 ; DOI : https://doi.org/10.4000/ leportique. 2771

Ce document a été généré automatiquement le 12 avril 2021.

Tous droits réservés 


\title{
Débat Sons et Images
}

\author{
Philippe Lafosse, Jacques Drillon, Renato Berta, Benoît Turquety et Léon \\ Garcia Jordan
}

1 Francis Guermann - Voici l'immense débat... Je ne vous présenterai pas nos invités parce que Philippe Lafosse, qui l'animera, va le faire, mais je veux simplement et particulièrement le remercier lui, parce qu'il nous a été d'une aide précieuse pour l'élaboration de notre rétrospective et je lui laisse la parole ainsi qu'à nos invités...

2 Philippe Lafosse - Merci. Et commençons effectivement maintenant cette discussion, ce débat, sur musique, voix, sons dans le cinéma de Danièle Huillet et Jean-Marie Straub... Lorsqu'on parle de musique et voix, on pense avant tout à trois films, Chronique d'Anna Magdalena Bach en 1967, Moise et Aaron en 1974 et Du jour au lendemain en 1996. Mais la musique est là dès le début et c'est peut être aussi de cela dont nous parlerons cet après-midi. Sans citer tous les films, on peut rappeler qu'on entend Bach et des extraits de Transmutations de François Louis dès le premier film, dès 1962 avec Machorka-Muff, puis Bach à nouveau, et Bartók, avec Non réconciliés ou Seule la violence aide où la violence règne, leur deuxième film, tourné en 1964-1965. Bach encore, on vient de le voir, de l'entendre, avec la projection du Fiancé, la Comédienne et le Maquereau, film de 1968, et quatrième film de Danièle Huillet et Jean-Marie Straub. La même année que Introduction à la « Musique d'accompagnement pour une scène de film» de Arnold Schoenberg qu'on vient également de voir, 1972, on retrouve Bach avec Leçons d'histoire: la Passion selon saint Matthieu. Bach qu'on entend également dans De la nuée à la résistance en 1978, dans La Mort d'Empédocle en 1986 (La Mort d'Empédocle ou Quand le vert de la terre brillera à nouveau pour vous). Faisons un petit saut: en 1988, ce sera Noir Péché avec là Ludwig van Beethoven. En 1991, Antigone et la musique de Bern Alois Zimmermann. Trois ans plus tard, il y aura Lothringen! et Haydn puis, en 1998, Beethoven à nouveau dans Sicilia! Avant de retrouver Bach dans Ouvriers, paysans en 2000. Et pour finir, citons encore Varèse en 2002 dans Humiliés et, plus récemment, O somma luce. Bref, tout en privilégiant, comme je vous le disais, Chronique d'Anna Magdalena Bach, Moïse et Aaron et $\mathrm{Du}$ jour au lendemain, nous pourrons donc aussi discuter ensemble de ces autres films. Ensemble, c'est-à-dire avec... À ma gauche, Renato Berta: Renato Berta est chef opérateur comme on dit, mais il n'aime pas beaucoup qu'on l'appelle comme cela aussi on pourrait dire « homme de lumière », « homme de l'image »; il a travaillé depuis 1968 
avec Alain Tanner, Alain Resnais, Manuel de Oliveira, Daniel Schmid, Jean-Luc Godard, Amos Gitai, et comme on est obligé de faire des sélections, j'avais fait une petite liste à laquelle il a ajouté Louis Malle; et donc avec Danièle Huillet et Jean-Marie Straub, une collaboration qui est peut être (c'est lui qui pourrait dire) la plus importante puisque, en gros, c'est plus ou moins quatorze films, et, quand je dis plus ou moins, c'est que ça commence avec Othon, sur lequel il était assistant, et continue avec Leçons d'histoire, Introduction à la "Musique d'accompagnement pour une scène de film " d'Arnold Schoenberg, Moïse et Aaron (il va dire non parce qu'il n'a travaillé que sur les plans en Égypte), puis Fortini/Cani, La Mort d'Empédocle, Ouvriers, paysans, Humiliés.... Une visite au Louvre, Ces rencontres avec eux, jusqu'aux derniers 0 somma luce et Joachim Gatti. À côté de Renato, Jacques Drillon : vous le connaissez peut-être parce qu'il est déjà intervenu ici ; Jacques Drillon est ancien producteur de radio, linguiste, enseignant de linguistique, de stylistique, journaliste au Nouvel Observateur, grammairien, traducteur, notamment de L'Art de la Fugue, dernière ceuvre de Bach pour Clavecin de Gustav Leonhardt, il est l'auteur d'une quinzaine d'ouvrages traitant de musicographie et en particulier de De la Musique et de Sur Leonhardt parus respectivement en 1998 et 2009 aux éditions Gallimard dans la collection L'Infini, ce dernier étant dédié à Jean-Marie Straub et, pour faire plaisir à Jean-Marie qui est là-haut, je citerai aussi qu'il est l'auteur de Charles d'Orléans ou le Génie mélancolique en $1993 \mathrm{chez}$ Lattès. Au bout de la table, sur la droite, Benoît Turquety : Benoît est critique, il a été enseignant à l'école Nationale Louis Lumière et à Paris VIII Vincennes; il est actuellement enseignant à l'université de Lausanne et l'auteur notamment de Danièle Huillet et Jean Marie Straub "objectivistes » en cinéma paru aux éditions de l'Âge d'Homme en 2009. Et puis, à ma droite, León García Jordán : photographe, réalisateur, il prépare actuellement un film, À travers la Chacone, avec Hopkinson Smith, inspiré par Chronique d'Anna Magdalena Bach et il participe aux sous titrages des films Straub-Huillet pour l'édition DVD espagnole, édition qui a déjà fait paraître Chronique d'Anna Magdalena Bach, Moïse et Aaron, Introduction à la «Musique d'accompagnement pour une scène de film» de Arnold Schoenberg, Du jour au lendemain, quatre coffrets en tout; il nous en parlera car son expérience sur le sous-titrage en castillan de Chronique... est très intéressante.

3 León García Jordán - Je vous présente Philippe Lafosse qui, outre le fait d'être à l'origine de l'édition espagnole qui vient d'être citée et de s'en occuper, est chargé de l'édition en DVD des films de Jean-Marie Straub et Danièle Huillet en France, aux éditions Montparnasse, qui comprend déjà plus de vingt films en cinq coffrets DVD ; il est aussi l'auteur du livre L'Étrange cas de Madame Huillet et Monsieur Straub/Comédie policière avec Danièle Huillet, Jean-Marie Straub et le public, paru chez Ombres en 2007, et de Maintenant dites-moi quelque chose publié en janvier 2011 chez Scribest/Al-prod; il a également réalisé le long métrage Dites-moi quelque chose à partir des débats avec le public pendant la rétrospective des films de Jean-Marie Straub et Danièle Huillet qu'il organisa au Reflet Médicis à Paris en 2007 et 2008.

P. L. - Merci León. Maintenant que tout le monde est présenté, alors musique et sons... On sait qu'à propos du son et de l'image qui se doivent d'être indéfectiblement liés, Jean-Marie Straub évoque souvent Renoir, vous l'avez peut-être entendu ici même: "Renoir, dit-il, il prenait un cendrier, le laissait tomber sur le sol et puis il disait "Vous voyez, ce bruit-là et ce mouvement-là sont liés". Eh bien moi, continue Jean-Marie Straub, que je cite mot pour mot, je n'ai aucune religion, mais j'ai cette religion-là, ça s'appelle le direct. Le cinéma, c'est ça, à partir du moment où on a inventé le son il n'y a aucune raison de trafiquer, on peut en rajouter, on peut en enlever, mais il n'y a aucune raison d'enregistrer séparément deux 
choses qui ont eu lieu en même temps, sinon c'est ridicule et ennuyeux». Pour les Straub, le cinéma c'est du concret, c'est du direct, c'est de la matière et cela, du début à la fin. Les films sont fabriqués, construits pour porter à la connaissance des œuvres, des musiques, des textes, des compositions, à la connaissance de spectateurs qui, les ignorant ou les connaissant mal, les découvrent pleinement, car, comme dit Jean-Marie, ces films ne sont pas en priorité pour ceux et celles qui ont étudié Corneille à l'université et Moïse et Aaron, ils sont d'abord pour ceux et celles qui pensent que Schoenberg est strident, voire impossible, et s'ils découvrent ces œuvres, ces textes, ces musiques, s'ils deviennent pour eux, spectateurs, de la mémoire vive, en quelque sorte, alors que souvent ils en avaient été écartés ou s'en étaient tenus à l'écart, le principe de ce cinéma-matière, ce parti pris, cet engagement sans cesse affirmé, y est pour beaucoup. Il est ce qui fait l'incarnation, la vie tout simplement, il est ce qui fait que les films existent. Ce principe du cinéma-matière où tout vibre, tout est incarné, les lieux, la lumière, les couleurs, et le reste, donne aux plans, aux films, une intensité, une force, une sensualité, qui participent beaucoup de la résistance, voire de leur subversion, et qui, par les contraintes, permet d'accueillir jusqu'à l'émerveillement tout ce qui arrive, tout ce qui surgit. Le travail de la matière cinématographique est ce qui donne des objets uniques. C'est de ce travail et de cette unicité dont nous allons essayer de parler aujourd'hui avec au cœur la musique, les sons, la voix... Je passerai la parole à Jacques Drillon en lui demandant de nous parler de cette unicité dans Chronique d'Anna Magdalena Bach, de ce qui fait que ce film est unique...

Jacques Drillon - Pardon, je ne vais pas du tout répondre à votre question. Je voudrais que vous relisiez dans la précédente feuille, la déclaration de Straub sur le son direct...

Philippe Lafosse - « Renoir, il prenait un cendrier, le laissait tomber sur le sol et puis il disait » Vous voyez, ce bruit-là et ce mouvement-là sont liés ». Eh bien moi, je n'ai aucune religion, mais j'ai cette religion-là, ça s'appelle le direct. Le cinéma, c'est ça, à partir du moment où on a inventé le son il n'y a aucune raison de trafiquer, on peut en rajouter, on peut en enlever, mais il n'y a aucune raison d'enregistrer séparément deux choses qui ont eu lieu en même temps, sinon c'est ridicule et ennuyeux ».

7 J. D. - Je vous ai demandé de relire cet extrait parce que je m'étonne. Je m'étonne et, Jean-Marie, puisque vous êtes là, je voudrais que vous expliquiez la présence de ce chœur de Bach au début du Fiancé, la Comédienne et le maquereau que je viens de découvrir. Je voudrais que vous expliquiez la présence de la musique de film dans ce film, car je ne comprends pas... Mon avis personnel n'a aucune importance, mais je suis résolument contre la présence de la musique au cinéma, si ce n'est la musique qu'on voit sur l'écran comme disait Bresson juste avant d'ajouter : plus de musique du tout. Je pense qu'à l'exception de quelques glorieux cas où c'est fait avec une telle intelligence qu'on finit par la supporter, la musique est un artifice extrêmement facile, extrêmement paresseux. Je pense que dans presque tous les cas la musique est utilisée d'abord parce qu'on peut l'utiliser, comme on construit un Airbus de huit cents places parce qu'on peut le construire même si on n'en a pas besoin. Il me semble que le cinéma peut très bien se passer de la musique. S'il ne s'en passe pas, c'est que quelque chose manque au film. Je reviens donc à ma question à Jean-Marie : on a entendu un chœur de Bach mis sur une image, et je voudrais que vous justifiiez la présence de cette musique qui n'a pas été enregistrée en même temps que le reste. Un son a été enregistré dans un studio avec un orchestre, un chanteur, des chœurs, etc. puis on a mis ça sur une image : ça me semble un peu contradictoire avec le fait de dire « on ne trafique pas le son, on 
prend le son qui existe, les gens parlent, on les enregistre, ça a lieu en même temps, on le fait en même temps ».

Jean-Marie Straub - Je n'ai jamais été un homme conséquent, la conséquence ça me fait une peur bleue, ça aboutit au nazisme. Alors je me laisse aller à des contradictions, c'est tout. Mais maintenant, par ailleurs, cette musique qui intervient là, c'est justement le son qui arrive après le muet, c'est tout. Il se trouve que c'est de la musique. J'ai souvent dit que, même dans des chefs d'œuvre, de John Ford ou d'autres, quand tout d'un coup une musique venait perturber le film et que ces films avaient du succès grâce à cette musique, c'était de la démagogie : nous sommes d'accord, je n'ai pas besoin d'insister. Mais là, c'est le sentiment qu'on a eu en découvrant cette rue-là, quatre ou cinq ans avant de tourner le film, en revenant de la périphérie de Munich où on allait voir des films. Il n'y avait pas de tramway pour aller là-bas, il n'y avait pas de tramway pour en revenir. On revenait à pied en pleine nuit, voilà. Et on a découvert cette rue-là parce que c'était la ligne droite pour rentrer chez nous à pied. Et alors là, on a éprouvé certains sentiments comme tout le monde éprouve des sentiments devant quelque chose qu'il voit. Quand, un beau jour, on a eu l'occasion de faire ce film, provoqué par monsieur Fassbinder, on a filmé cette rue seulement deux fois avec un "farway bus", avec deux caméras à chaque fois, une caméra à plat et l'autre avec un petit angle, une petite diagonale, deux fois seulement, et la deuxième fois ça devenait très dangereux parce qu'ils voulaient nous crever les pneus - j'entends les braves gens qui s'appellent les maquereaux, dont c'est le gagne-pain, comme on dit. Donc, on avait quatre prises et ça nous suffisait amplement. On s'est barré. Et puis on a choisi une de ces quatre prises. Alors, on avait une réalité, là, quelque chose, un poids, et puis cette musique-là, c'est une contradiction à ça! C'est tout! Le texte de cette musique, c'est la clé de votre question, dit: "Du tag! Oh jour! Wann werst du sein? Quand seras-tu? Quand viendrastu ?...», etc. C'est l'impatience, pour être très prétentieux, c'est l'impatience de la révolution. Point final. Bach nous sert à ça. On n'a pas du tout enregistré cette musique pour ce film, on l'a piquée à Gustav Leonhardt, et ce n'est pas du tout un chœur, c'est un choral final de l'Oratorio de l'Ascension.

9 J. D. - Simplement, j'ai toujours l'impression qu'à partir du moment où on met une musique sur une image, c'est qu'on veut nous dire ce qu'on doit éprouver, c'est qu'on veut forcer les spectateurs à éprouver un sentiment complètement dirigé, un peu comme on focalise une lumière. Et quoi que vous fassiez, si vous mettez cette musique sur cette image...

10 J.-M. S. - Mais c'est une violence, c'est une violence !

11 J. D. - Non, pas simplement : ça donne un lyrisme à l'image, ça lui donne de l'ampleur.

12 J.-M. S. - Ça la contredit !

13 J. D. - Oui, ça la contredit dans votre esprit, éventuellement, mais est-ce le cas pour les spectateurs? Est-ce que, pour les spectateurs, ça ne donne pas simplement de la grandeur à l'image ? Est-ce que ça montre la petitesse ? Voilà la question. C'est comme si le spectateur ne pouvait...

14 J.-M. S. - Ça montre la grandeur de la misère humaine et de la misère sociale !

15 J. D. - On peut dire ça comme ça... Mais, prenons par exemple la fin d'Un condamné à mort s'est échappé. Quand Fontaine et Jost, les deux personnages, ont réussi à passer le dernier mur et se retrouvent dans la rue, le gamin, Jost, dit à Fontaine «Si ma mère me voyait », et on entend aussitôt la messe en ut mineur de Mozart. "Si ma mère me voyait », 
c'est une phrase fantastique, on est complètement bouleversé, ils ont enfin réussi, ils sont libres, on est submergé par une espèce de vague de bien-être, et tout d'un coup on entend la messe de Mozart...

J.-M. S. - On l'a déjà entendue bien des fois au cours du film, quand ils vont verser leur merde et leur urine...

J. D. - Oui, oui, je suis d'accord, mais le fait est que, sur cette scène-là, j'ai l'impression que Bresson ne nous a pas laissés éprouver la joie qu'on a de les voir libres, parce qu'on éprouve une joie, une vraie joie avec un grand J si on peut dire, et qu'il nous dit, avec cette musique, "c'est bien de la joie que vous devez éprouver maintenant, ne vous trompez pas ». Avec cette musique sublime qui arrive tout d'un coup, c'est comme si on avait un plan d'hélicoptère, c'est comme si on s'écartait du sol, comme si on montait au ciel. Donc, soit il fallait monter dans un hélicoptère et filmer d'en haut, soit on restait en bas, mais ce n'était pas la peine de filmer d'en bas en nous disant ce qu'on doit être en haut. J'ai l'impression qu'avec la musique on cherche toujours à en rajouter quand l'image n'a pas suffisamment de force. On ajoute de la musique car c'est un art hyperpuissant - voir ce fameux livre de Pascal Quignard, La Haine de la musique -, si puissant qu'on l'a réservé parfois aux derniers usages, y compris à Auschwitz, où on lui a fait faire ce qu'on voulait, parce qu'elle peut tout faire, la musique, elle peut absolument tout faire, elle peut vous faire éclater d'énergie, elle peut vous écraser de déprime, elle peut... La musique a un pouvoir à la fois intellectuel, moral, physique, esthétique, etc. Ça rentre dans le ventre, ça entre dans l'âme, ça vous transforme, ça vous modèle comme de la glaise. Et on utilise la musique parce qu'elle a le pouvoir de faire cela.

P. L. - Renato, tu veux bien réagir à ce que dit Jacques.

Renato Berta - Ça m'est arrivé lors d'un tournage avec un metteur en scène, s'il vous plaît ne me demandez pas son nom, qui était très sympathique. Il s'agissait de faire un mouvement de caméra assez compliqué. Je le fais, c'était un plan long, et vers la fin, alors que tout était bien, hop il y a eu un petit accroc, de sorte que techniquement ce n'était pas très bien. Et le metteur en scène me dit: «Ne t'en fais pas, je mettrai de la musique et ça passera ». Voilà.

P. L. - Garde le micro, Renato. J'aimerais bien que tu nous parles de la façon dont tu travaillais, en prenant des exemples précis peut-être, avec Danièle et Jean-Marie. Dans Introduction..., par exemple, la musique de Schoenberg dure une dizaine de minutes: quand tu as tourné savais-tu qu'il y avait cette musique de Schoenberg sur des plans? Autre exemple : 0 somma luce, qui commence par un petit peu plus de sept minutes de Désert de Varèse, sur du noir, tant et si bien que le premier plan éclate et éblouit... Quand tu as tourné ce premier plan, savais-tu qu'il allait y avoir ce noir avant et cette musique de Varèse?

R. B. - Et moi, très lâchement, j'ai envie de retourner ces questions à Jean-Marie. JeanMarie, c'était déjà prévu d'avoir du Varèse avant ?

J.-M. S. - Non, pas du tout, c'était impossible...

R. B. - Alors voilà, ce n'était pas prévu, c'était impossible, et donc je ne le savais pas. Le petit Schoenberg, lui, s'est fait... sans que je le sache avant... On tournait Leçons d'histoire et ça a été un peu un film piraté dans le plan de travail, c'est-à-dire que tout à coup - je t'assure que je n'étais même pas au courant - je vois Jean-Marie se mettre sur un mur et commencer à répéter. Je lui dis : «Tiens, on change de film?». Et il me dit oui. «Ah bon 
d'accord, très bien ». Ça s'est fait à peu près comme ça, je savais à peine que ce film était au feu. Je me trompe, Jean-Marie? pouvoir parler autant de musique que du cinéma de Straub-Huillet et de l'aspect visionnaire de ce projet qui est le premier de leur œuvre puisqu'il s'origine dans les années 1950, à une époque où se produisait un changement important dans la reconstitution de la musique. Rappelons que c'est en 1952 que Gustav Leonhardt publie à Vienne sa thèse sur L'Art de la fugue, et que, deux ans après, Jean-Marie Straub découvre L'Art de la fugue, la musique, avec son ami organiste François Louis... À cette même époque, en 1953 précisément, Nikolaus Harnoncourt et sa femme, Alice Hoffelner, fondent le Concentus Musicus à Vienne et rencontrent Alfred Deller qui était alors un peu plus âgé mais qui commençait aussi à emprunter une voie qui s'était perdue - et, comme vous l'avez dit vendredi, Jean-Marie, votre idée était de remonter le courant avant Mendelssohn. Alfred Deller travaillait sur la voix de haute-contre qui avait disparu à partir de Mendelssohn justement et découvrait une chose qui allait être certes très importante pour la musique, pour l'interprétation de la musique ancienne dans la deuxième moitié du vingtième siècle, mais qui allait rayonner aussi bien au-delà à savoir dans le travail du texte, de diction du texte, et dans la façon de considérer celui-ci comme de la matière. Cette voie que défrichera Alfred Deller, Gustav Leonhardt la décrit bien quand il dit que son maître véritable est Alfred Deller car il lui a appris l'éloquence au clavecin et que l'intelligibilité et le poids des mots étaient plus importants que la voix - le poids des mots, ça remonte à Heinrich Schütz et à JeanSébastien Bach, et on ne parle pas ici du "Sprechgesang», le chanté parlé, de Schoenberg, mais cela vient de là : la musique au cœur du texte, le sens des mots, des mots intelligibles. Donc, alors qu'il y a ça dans le domaine musical, cette effervescence des années 1950, Straub démarre son projet, il découvre le disque que Leonhardt a enregistré après la publication de sa thèse, L'Art de la fugue; un scénario et un découpage sont élaborés à partir des œuvres de Bach, des textes des partitions, et envoyés à Leonhardt. Après, on le sait tous, le film prendra dix ans à pouvoir se tourner à cause d'une question de production mais c'est comme premier projet cinématographique qu'il est important de voir ce travail parce qu'il y une déclaration d'intention cinématographique rivée à ces années-là...

P. L. - Concernant le traitement de la musique, des voix, des textes qu'ils soient de Heinrich Böll, Franz Kafka...

31 L. G. J. - Exactement. Le traitement de la musique, du texte, comme un artisanat, un artisanat qui fera, à partir de ce moment, du cinéma Straub-Huillet un cinéma d'artisan, un cinéma fait toujours avec la même pureté, une "pureté terroriste » comme le dit Alberto Moravia à la sortie de Chronique... Ce cinéma-là, qui va advenir à partir de 
Machorka Muff, est un cinéma pur et primitif, comme celui des opérateurs Lumière. C'est le même travail - rappelons aussi que Stockhausen avait dit à l'époque que Machorka Muff était un film musical, et qu'au début de ce premier film est écrit: "Un rêve symboliquement abstrait, pas une histoire »; l'art cinématographique ne raconte pas d'histoire, c'est autre chose que le narratif, le récit, tout est déjà dans cette déclaration d'intention. Il y a une belle émission, réalisée je crois par Éric Rohmer, où dialoguent Henri Langlois et Jean Renoir en regardant les premières prises de vue des opérateurs Lumière, L'Arrivée d'un train en gare de La Ciotat, par exemple, qui date de 1895, et où on perçoit, grâce à leurs commentaires, combien il y avait une science des plans : les plans devaient être tournés après beaucoup de repérages pour savoir où placer la caméra afin de capter le plus grand nombre d'événements en tenant compte de la fixité obligatoire de la caméra, de la durée limitée des bobines, et encore cela ne concernait-il évidemment que l'image... Quand je parle de cinéma primitif, de film manifeste de pureté, qui définit les tâches du cinématographe, c'est dans ce sens-là ; c'est ce travail qui va être fait avec la musique pour Chronique..., de façon à ce que l'image et le son, le cinématographe, cette "science des plans ", se mettent au service de la musique pour la rendre de la manière la plus pure et pour que le spectateur ait avant tout une expérience musicale, pour qu'il découvre bien entendu la musique de Bach mais qu'il ait surtout la possibilité de découvrir ce qu'est une matière cinématographique, cela allant jusqu'à voir des gens en train de réaliser un travail devant la caméra, en l'occurrence le travail musical de Gustav Leonhardt et des musiciens, un artisanat fait avec le corps, d'où l'extrême sensualité du film, comme d'ailleurs de toute la filmographie de Straub-Huillet.

P. L. - Jacques Drillon, Chronique... c'est l'histoire d'une rencontre...

J. D. - Disons que les deux projets coïncidaient, d'une part celui de Leonhardt, qui était de partir de données historiques exactes et d'un instrument, surtout, qui impose sa loi, et d'autre part celui de Straub qui voulait montrer exactement comment est la chose, comment est la musique quand on la fait vraiment. Oui, il me semble que ces deux projets étaient... destinés, au sens figuré, à se rencontrer, à se compléter. Je pense que l'un, Leonhardt, s'est dit qu'il allait écouter ce qu'était un clavecin, puisque c'était l'instrument qu'il avait véritablement redécouvert, écouter ce que le clavecin lui disait, lui imposait, comme une didascalie d'auteur dramatique, ce qu'il lui dictait, comme quand vous voyez un muet essayer de vous dire quelque chose avec les yeux; je pense qu'il a tâché de comprendre ce que voulait le clavecin, comment il voulait être joué, et essayer de comprendre aussi le texte de la musique de Bach, ce qu'il demandait ; bref, je crois qu'il a senti là l'occasion de se mettre de façon inédite à l'écoute de la matière existante, à l'écoute c'est-à-dire sans idées préconçues sur l'instrument, les textes, dont les textes musicaux, les traités, tout ce qui est porteur de réalité historique. Et je pense que l'autre, Straub, a été dans le même état d'esprit du côté de l'image, de l'écoute, de l'enregistrement, du cinéma. D'ailleurs, on peut rappeler que Danièle Huillet et JeanMarie Straub ont décidé de contacter Leonhardt uniquement après avoir entendu les disques; ils ne savaient pas qu'il avait cette tête de Christ de Greco descendu de sa croix, ils ne savaient pas qu'il était beau, noble et aristocratique; c'est en écoutant qu'ils se sont dit que cet homme parlait le langage vrai de cette musique-là, sans s'effacer. La grande difficulté de l'interprétation musicale est là : si on joue seulement le texte, on lui casse la gueule, on le trahit; si on en ajoute, on le trahit aussi. Il faut trouver une sorte de juste poids : un maximum de poids, mais un maximum de poids exact, pour que le texte lui-même soit juste... À ce propos de langage vrai et de justesse, 
Leonhardt lui-même a du reste rendu hommage aux Straub quand il a confié des années après qu'ils n'étaient pas des musiciens mais qu'ils avaient pourtant entendu ça. Entendu quoi, compris quoi? Compris qu'avec lui ils allaient pouvoir faire quelque chose parce qu'ils avaient senti qui il était, qui était la personne qui jouait, quelqu'un qui ne cherchait évidemment pas à s'interposer avec le texte mais qui ne s'effaçait pas non plus complètement, car il n'y a pas de raison de s'effacer - on peut être aussi orgueilleux quand on est musicien -, quelqu'un qui investissait la bonne somme au bon endroit au bon moment, et qui tenait compte des réalités historiques, musicales, " solfégiques ", stylistiques, etc.

R. B. - Dans un cas pareil, je me demande comment peuvent s'équilibrer d'un côté la partie jeu, la partie comédie, celle du comédien, et de l'autre l'instrument...

J. D. - L'instrument lui-même a une telle prégnance, il est tellement premier, tellement fort comme objet, fait dans une certaine matière, d'une certaine forme, avec un timbre donné, un son déterminé, une mécanique, etc., que c'est lui le patron. Au départ, c'est l'objet le patron, c'est lui qui dit: "Vous voulez tenir une note sur un clavecin? Vous ne pouvez pas? Forcément : c'est court, c'est tout! Alors débrouillez-vous». Leonhardt, lui, répond: "Voyons, le son du clavecin est court, tout le monde essaie de l'allonger le plus possible en allant jusqu'à fabriquer des clavecins plus puissants, tenant plus le son, mais moi je n'allongerai pas le son du clavecin, je le jouerai encore plus bref ». Si le clavecin est bref c'est qu'il aime le bref. Leonhardt va donc piquer les notes, au sens de les jouer très courtes, et personne ne pique comme lui, il procède un peu comme les couturiers quand ils ont une femme très grande à habiller: ils ne cherchent pas à l'habiller en pot à tabac, ils cherchent à l'habiller en femme grande - en la chaussant de hauts talons, et ils ont raison. C'est comme ça qu'il faut faire, il faut prendre les choses comme elles sont. Aussi, Leonhardt a pris le clavecin comme il est, il a respecté cette espèce de tyrannie de l'instrument, ou de loi qu'il impose, et une fois qu'on respecte ce que l'instrument vous dicte comme une phrase exige ses signes de ponctuation - une phrase demande à être ponctuée d'une certaine manière, vous avez le droit de la ponctuer comme vous voulez, mais elle ne sera pas contente -, on s'aperçoit que, comme par hasard, il n'y a pas tant que ça à rajouter : si on respecte l'instrument, on fait déjà cinquante pour cent du travail. Ensuite, Leonhardt s'est dit qu'il respecterait le texte s'il jouait ce qui était écrit, lui qui savait lire et avait appris le solfège de cette époque-là, ce que les autres ne savaient pas. Glenn Gould, et Dieu sait que je l'aime et lui tire mon chapeau, a joué sur les éditions Schirmer qui sont absolument dégueulasses, pleines de fautes, il a joué un texte qui est fautif et en ignorant le solfège du dix-huitième siècle : en conséquence, il faisait sans cesse des fautes. Leonhardt ne faisait pas ces fautes-là, de texte, de solfège, de style, de temps. Autre exemple de faute : si on fait un menuet à la vitesse à laquelle le faisait Karajan, et cela depuis Mendelssohn, les gens qui sont censés danser sont obligés d'attendre en l'air avant de retomber; ce qui signifie qu'on faisait le menuet beaucoup trop lentement et qu'il fallait l'accélérer pour que les danseurs puissent le danser, tout simplement. Leonhardt a respecté ces choses-là, une chose, puis une autre, et une autre encore, et petit à petit, la fondation est devenue de plus en plus solide, la maison a commencé à se construire, et le travail d'interprétation proprement dit qu'il y avait à faire s'est réduit ; il restait dans une petite marge acceptable d'investissement personnel. Ce qui a donné une musique telle que les Straub, qui n'étaient pas musiciens, se sont dit en l'écoutant jouer L'Art de la fugue - en plus ce n'était pas n'importe quoi - : « C'est lui qu'on veut ! » 
P. L. - Benoît, Moïse et Aaron c'est aussi simple que Chronique... : on tourne en son direct, et en plein air cette fois-ci. Comment s'est précisément fabriqué ce film, comment s'estil élaboré ? Peux-tu nous éclairer, car ce n'est évidemment pas si simple que ça?

Benoît Turquety - Non, ce n'est effectivement pas si simple. De toute façon, le son direct ce n'est pas si simple, c'est une chose assez compliquée en fait. On en a déjà d'ailleurs le sentiment avec Le Fiancé, la Comédienne et le Maquereau qu'on a vu: on perçoit bien que, quand on prend du son direct, les décisions de son sont, par exemple, des décisions de décor. Si des gens jouent sur une scène avec un plancher en bois, comme dans ce film, ils produisent un certain son en marchant; le son des portes est aussi important dans la partie théâtre, il est sec, il joue un vrai rôle. D'ailleurs, ce que Jacques Drillon vient de dire me fait penser qu'il y avait une affinité d'oreille entre ce que faisait Leonhardt d'un côté et ce que faisaient les Straub: on voit bien que les Straub aiment aussi quand c'est piqué, quand c'est sec, quand ça va vite, quand ça va même parfois trop vite pour être vraiment suivi en terme de sens, de signification... Le cinéma des Straub a l'image d'un cinéma lent, presque austère, mais en réalité ça va très vite; il faut vraiment arriver à suivre. Je pense donc qu'il y a en commun un sens de la vitesse. Pour revenir à Moïse et Aaron, il y eut effectivement un choix crucial qui est d'avoir voulu tourner ce film en extérieur et en son direct - son direct parce que c'est non négociable et extérieur parce que c'était lié au projet dès le départ. Une autre décision, pas tellement négociable non plus pour eux et dont on n'a pas encore parlé, est celle d'enregistrer en son monophonique : le son chez Straub, c'est mono.

P. L. - On dit là aussi qu'ils refusent la stéréo parce qu'ils sont austères...

B. T. - Oui, c'est pareil, mais ce n'est pas là non plus une question d'austérité. Dans une salle, le son mono n'arrive pas de tout autour; disons que maintenant il arrive quand même de tout autour mais il est unique, et, dans les salles traditionnelles, il vient de derrière l'écran. Il y a donc un lien entre l'image et le son, lien qui n'existe pas avec la stéréo où un son vient d'un seul côté, un autre surgit de l'autre côté, etc. C'est vraiment un rapport à la question de l'espace, de l'espace qu'on filme et de l'espace dans lequel on est quand on filme, qui change la donne. En l'occurrence, pour Moïse et Aaron, ce choix a d'autres implications fortes. Par exemple, il y a dans la partition deux chœurs : l'un est le chœur-Dieu - quand le buisson ardent est là, on entend un chœur qui est la voix du buisson ardent : on peut se dire que c'est Dieu -, l'autre est le chœur-Peuple. Chez Schoenberg, il y a beaucoup de différences de matière, de construction, entre ces deux chœurs : il y a un chœur qu'on voit, qui est le peuple, qui est sur scène ou pas, et puis il y a un chœur qui peut être complètement hors-champ, le chœur-Dieu, qui a six voix de solistes par exemple qui, d'après la partition de Schoenberg, doivent venir de plusieurs endroits différents dans la salle, les six voix ne devant bien se retrouver que dans la salle - Schoenberg imagine même de le faire avec des téléphones ou des câbles. Avec la stéréo, on aurait pu rendre un effet de ce type-là, mais en mono c'est impossible. D'où le fait que dans le film le son du chœur qui est Dieu ne présente plus de différences avec celui du chœur qui est le peuple: cette différence-là, recherchée par Schoenberg, ne peut plus être rendue. C'est un élément très fort dans Moïse et Aaron et qui n'est pas du tout anodin; il produit au contraire un sens important car que déduire du fait que le chœur qui est Dieu et le chœur qui est le peuple produisent le même son, du fait que ces deux chœurs sont le même, en sont un seul? 
dessus : il faut choisir le lieu en fonction et ça a demandé à Jean-Marie Straub et Danièle Huillet de parcourir pas mal l'Italie pour arriver à trouver un lieu où on puisse jouer cette pièce de manière cohérente sans que ne se posent fortement des problèmes de son. Cela fait, Moïse et Aaron est un opéra, on va donc avoir des gens qui chantent et puis un orchestre, l'orchestre. Là, a priori, c'est un obstacle de plus, quand on veut tourner en extérieur ; il est difficile d'amener un orchestre et de le faire jouer pendant les plans, sachant que ça poserait un énorme problème de prise de son. Il est alors indispensable de prendre une série de décisions et une de celles-ci fut d'enregistrer d'abord l'orchestre, dans les studios de la radio de Vienne. Donc on enregistre l'orchestre d'un côté, puis on garde cette musique et on fait chanter des chanteurs dessus, mais les chanteurs, eux, vont chanter en extérieur, par conséquent il faut s'arranger - c'est en tout cas le parti qui fut pris - pour que le son ait à peu près la même qualité, le même type de réverbération, ce qui veut dire que là aussi ils ont décidé - et c'est là également une question de goût et d'oreille - d'enregistrer sec, c'est-à-dire sans réverbération parce qu'ils avaient vu, en faisant des tests avec leur ingénieur du son, que la qualité sonore de l'amphithéâtre et celle naturelle du studio d'enregistrement de l'orchestre étaient à peu près les mêmes et que donc en enregistrant sec, on retrouvait en quelque sorte les sons raccordés. Après, une fois sur place, le défi est d'enregistrer les chanteurs et que ceux-ci entendent en même temps la musique tout en enregistrant indépendamment chanteurs et musique et en enregistrant éventuellement chaque chanteur indépendamment l'un de l'autre - sinon on ne peut s'en sortir au mixage. Voilà qui demande un protocole extrêmement compliqué. Ainsi, il a fallu équiper les chanteurs d'oreillettes et utiliser plusieurs magnétophones sur le tournage, trois exactement dont l'un diffusait la musique enregistrée en studio dans les oreillettes des chanteurs, du côté évidemment où ils n'étaient pas dans le champ, où ça ne se voyait pas; les chanteurs avaient alors en face d'eux, mais pas trop près, la caméra et le chef d'orchestre qui les dirigeait, et ils devaient chanter, si possible de manière audible, même si il y avait du vent, le soleil dans les yeux, etc. Le travail de l'ingénieur du son va alors être d'arriver à capter ce chant en même temps que l'orchestre. C'est donc relativement complexe. Ça demande un protocole calculé à l'avance; ça demande d'avoir un découpage plan par plan fait évidemment lui aussi à l'avance, où la partie orchestrale a été divisée en séquences cohérentes avec la partition, séquences qui correspondent au découpage filmique. C'est donc un protocole d'une extrême complexité. On ne s'en rend pas forcément compte à la vision et à l'écoute de Moïse et Aaron mais c'est très complexe. implications liées au son direct.

B. T. - Il y a toutes les décisions qui vont avec. Qu'est-ce que ça veut dire de filmer ainsi en son direct? Ça veut dire qu'on considère que le son direct est important pour les gens qui chantent devant la caméra, mais l'est moins pour l'orchestre, dont on estime qu'il n'a pas à être enregistré pendant le tournage. Ce ne sera pas le cas pour Du jour au lendemain, film pour lequel Straub et Huillet prendront l'autre parti : tourner en studio avec l'orchestre dans la fosse et les chanteurs sur la scène, et à chaque fois qu'on dit 
" action » - ils ne disent pas " action » mais admettons -, à chaque fois qu'on dit « on y va ", l'orchestre commence, les chanteurs commencent, et l'orchestre et les chanteurs s'arrêtent à la fin du plan, tout le monde joue donc en même temps. C'est un parti pris complètement différent. Mais, pour revenir à Moïse et Aaron, il y a cette idée d'une différence de statut entre la parole et l'orchestre : quand on parle ou chante devant une caméra, il faut que ce soit enregistré en son direct, avec d'ailleurs des scènes qui du coup sont intéressantes à voir se déployer. Vous vous souvenez de la procession devant le Veau d'Or: une malade arrive devant lui et chante, elle est prise en son direct, puis des mendiants la suivent, dont on entend le chœur mais qu'on ne voit pas chanter, et là ce sont des chœurs enregistrés à Vienne. Les mendiants ne sont pas pris en son direct. Avec ce seul exemple, on comprend ce qu'impliquent concrètement certains choix : puisqu'elle doit chanter pendant la prise, la malade se doit d'être une chanteuse sachant lire une partition, sachant vocaliser, etc. ; alors que les mendiants sont, eux, des gens des environs, des paysans; en conséquence, leur statut, leur rapport à ce qui est montré, ne sont pas du tout les mêmes. Ainsi, quand ils enlèvent leur tunique pour la donner au Veau d'Or, on voit que ces gens ont un bronzage et on devine des teeshirts modernes; on voit aussi que les dames ont le réflexe de faire des génuflexions, des signes de croix devant ce Veau d'Or, ce qui est... On n'aurait jamais eu ça avec des acteurs ou des chanteurs professionnels, on n'aurait pas eu ce type de gestes qui sont eux aussi, il faut le dire, ce qui intéresse les Straub. Pour résumer ce point, choisir de prendre en son direct ou pas a de très nombreuses conséquences, jusqu'au type de personnes pouvant ou non interpréter, «tenir le rôle ", en passant notamment par la lourdeur technique, par l'incidence sur les décors, par le choix des micros et de la direction dans lesquels on les met - ce qui change tout car on n'entend pas la même chose en fonction des micros et des directions choisies -, par les questions de statut pour le spectateur, par celles de matière sonore - ce n'est évidemment pas la même chose de voir et entendre les gens qui chantent dans l'instant, d'avoir Louis Devos qui chante contre le vent, qui chante avec le soleil dans les yeux, que d'entendre une voix prise en studio plaquée sur un chanteur, même si celui-ci a réalisé son propre doublage. Les Straub fonctionnent en son direct, c'est vrai, mais il est essentiel de voir les choix et les partis pris complexes qui en découlent.

P. L. - León, à propos de Chronique d'Anna Magdalena Bach et du son mono qu'a évoqué Benoît....

L. G. J. - Dans Chronique.., c'est une façon aussi de fabriquer l'espace des lieux dans lesquels la musique est jouée. À la différence de tous les Straubfilms, et en particulier de Moïse et Aaron et Du jour au lendemain, où on est dans un découpage par personnage, par acte, en fonction des nécessités de l'opéra, Chronique... est un film où le découpage est fait par la musique, comme si la musique était le personnage. À ce titre, du point de vue de la construction de l'espace, le choix de la prise de son monophonique est important. Si on prend le plan de la Passion selon saint Matthieu, ce long plan fixe sur tous les musiciens avec le premier chœur au premier plan et le deuxième chœur au fond, il n'y a, du fait du son mono, qu'une seule matière, et parce qu'il y a alors un chœur qu'on entend plus fort au premier plan que l'autre qui lui répond à l'arrière, on découvre la pensée musicale de Bach. Grâce au son mono, le spectateur a la possibilité de pénétrer la pensée musicale de Bach - qui se définit aussi par l'espace où cette musique était jouée. 

mais moi je dirais qu'on a aussi une sorte de stéréo spatiale. On n'a certes pas la stéréo spatiale gauche/droite, mais on a la stéréo proche/lointain, qui est une stéréo qui se fond dans le mono, qui peut exister à l'intérieur de la monophonie; et, dans le plan dont vous parlez, c'est encore accentué par le fait que ceux qui sont proches sont de dos alors que ceux qui sont le plus loin sont de face. On obtient vraiment un effet stéréo et cela par une économie de moyens. Là, c'est le cinéma qui fait la stéréo et non le son ; il n'y a pas le côté grand spectacle avec un haut-parleur ici, un autre là, ce dont, on peut le dire, rêvait tout de même Bach, qui ambitionnait de retrouver la musique vénitienne, avec des échos dans Saint-Marc. Là, c'est vraiment fait avec des moyens cinématographiques purs. Et, pour rester sur ces questions de matière sonore, en entendant Benoît Turquety expliquer la différence de matière entre les deux chœurs dans la partition de Schoenberg, je me disais que c'est un problème que Bach n'a, lui, absolument pas résolu. Il y a deux chœurs dans la Passion selon saint Matthieu, mais il ne résout pas le problème de savoir " d'où on parle ", comme on disait en 1968, c'est-à-dire qu'on entend le même son pour les deux ; et dans la Passion selon saint Jean c'est encore pire, on entend le même son de chœur pour la foule - qui, par exemple, demande la crucifixion - et l'assemblée qui interprète un chant religieux, comme si c'était le même personnage. Donc, dans cette histoire, Bach se prend les pieds dans le tapis. Ce n'était pas un auteur d'opéra, à un chœur il n'a pas attribué un personnage, il n'a pas su faire ça. Dans la Passion selon saint Matthieu, il est étonnant qu'il ne se serve pas des deux chœurs dans un but de personnalisation psychologique, si on peut dire; il ne s'en sert que pour obtenir des effets de stéréo. Dans la Passion selon saint Matthieu, un chœur aurait pu faire la foule méchante demandant qu'on crucifie Jésus, et un autre chœur l'assemblée. Mais non, c'est la foule partout. C'est la foule qui change de couleur, d'une manière très mal fichue, pas aboutie... présence du peuple dans Moïse et Aaron?

B. T. - Dans un entretien de l'époque de Moïse et Aaron, Jean-Marie Straub dit effectivement que le Buisson Ardent c'est le peuple hors champ. Ça a l'air d'être une formule, mais l'égalisation des chœurs, le fait que tout à coup on n'a plus de différence de matière audible entre eux, fait qu'à un moment peuple et Dieu se confondent. Et cela est accentué par le découpage : on entend d'abord ce chœur du Buisson Ardent dans la première scène hors champ, puis lorsqu'on le réentend il est à nouveau hors champ et il y a tout d'un coup un panoramique qui nous fait découvrir que ce chœur-là... c'est le peuple - cette fois-ci, ce n'était pas Dieu, c'était le peuple. Du coup, rétrospectivement, on comprend le premier chœur comme étant aussi le peuple. À l'époque, Straub disait aussi que Moïse et Aaron était_un film sur le peuple et sur la présence du peuple en politique - sur la naissance du peuple, en l'occurrence d'Israël. On est toujours dans l'idée que Dieu égale le peuple. Je ne sais pas si c'est ce que Schoenberg avait en tête, mais c'est là que le film se situe, et notamment par cet effet de monophonie qui est vraiment fort, effectivement, et qui travaille la spatialisation, qui joue sur le timbre aussi - c'est surtout frappant dans les champs contrechamps quand on filme deux personnes. 
première, ce qui est encore une décision forte. Les Straub pourraient, auraient pu faire tourner le micro, ils ont décidé qu'on entendrait le " détimbrage » et qu'après, quand on aura le contrechamp sur cette seconde personne qui sera alors dans le champ, le changement de timbre serait très important pour le spectateur. Ce qui n'est possible que dans le choix d'une spatialisation mono - en stéréo, il n'y a pas de "détimbrage ", la personne à gauche parle à gauche.

51 J. D. - On voit le chemin qui reste à parcourir aux gens d'opéra qui font des opéras filmés, qui sont quotidiennement aux prises avec ces questions-là mais qui, ne sachant pas y répondre, se mettent, et nous mettent, dans des situations grotesques : on entend comme s'ils étaient tout près des personnages qu'on aperçoit à deux cent cinquante mètres, parce qu'ils ont été enregistrés en studio à vingt centimètres du micro.

P. L. - Sur ce chemin, il y aurait des étapes comme voir Chronique d'Anna Magdalena Bach, Moïse et Aaron et d'autres, voir leur travail du son, la spatialisation, réfléchir aux enjeux de tel découpage, de la mise en scène, à telle ou telle étrangeté, analyser...

53 B. T. - À propos d'étrangeté... La répartition de l'espace dans la mise en scène de Moïse et Aaron est claire, mais... Je vais prendre la troisième scène, la dernière, de l'acte I où on a d'un côté le peuple et, en face, Moïse et Aaron, selon la grande ligne de l'ellipse, puis de l'autre côté, au milieu, sur l'autre axe, le prêtre des anciens dieux et trois convertis qui lui font en face. À un moment la caméra est au milieu de tout ce monde, vraiment pratiquement au milieu de l'ellipse, et elle va de l'un à l'autre, elle fait des panoramiques filés de l'un à l'autre: c'est quand même une drôle de manière de découper cinématographiquement. J'ai essayé de comprendre pourquoi ces panoramiques, comment ça fonctionnait, et je suis arrivé à la conclusion que l'intérêt de ce dispositif est que non seulement le spectateur assiste à une mise en scène de l'opéra dans laquelle il y a de l'action, du suspense, des personnages incarnant des problèmes, des idées, etc., mais qu'en plus, en même temps, il voit une analyse de cet opéra. En effet, ce découpage, a priori étrange, ne cesse en fait de mettre à jour et d'expliciter des rapports de force, d'expliquer les enjeux de pouvoir entre les personnages, de sorte qu'on est finalement amené non seulement à suivre l'action comme un spectateur lambda dans un film de John Ford, mais aussi à pouvoir comprendre les rapports de force qui se construisent et se modifient au fur et à mesure du film. Et cela simplement avec ce parti pris, avec ce dispositif assez simple, dont tout le monde pourrait s'emparer, réalisateurs d'opéras ou pas, qui consiste avoir ces quatre pôles qui restent immobiles à l'intérieur du champ tandis que la caméra ne cesse d'aller et venir pour désigner qui joue avec qui, qui joue contre qui, qui se bat, ou qui va avec tel ou tel pôle.

P. L. - Merci, Benoît. Y a-t-il des questions dans la salle sur des points qui ont été abordés ou d'autres, comme vous le souhaitez.

Spectateur - J'aurais une question pour Monsieur Drillon. Vendredi soir, vous avez habillé Karajan pour l'hiver, expliquant pourquoi il ne fallait pas ajouter des instruments supplémentaires pour rendre un effet. Sur le coup, j'étais à la fois sidéré et convaincu, donc je n'ai pas posé de question, je ne suis pas allé plus loin. Mais, après y avoir repensé, et sachant que je ne connais rien à la musique, ne peut-on pas dire que Karajan a essayé, avec ces moyens-là, de faire ressentir au spectateur d'aujourd'hui, plus environné de musique et avec une autre oreille, ce que ceux de l'époque, plus vierges et avec une autre écoute, ont ressenti quand ils ont entendu cette musique pour la première fois? Je crois que, pour Aïda, Verdi voulait prendre des instruments d'époque égyptiens, mais qu'en entendant leur son il a trouvé ça terrible et s'est dit 
que, si les Égyptiens de l'antiquité étaient impressionnés quand ils entendaient ces instruments, un auditeur de 1870 ne le serait pas parce que Berlioz et d'autres, Wagner peut-être, étaient passés par là, moyennant quoi, au lieu de prendre des instruments d'époque, il a choisi des modernes, non pas pour faire du toc mais pour essayer de s'approcher d'un ressenti. C'est un peu la même idée...

J. D. - Je comprends ce que vous voulez dire. En l'occurrence, si, dans sa Cinquième Symphonie, Beethoven avait quatre contrebasses, c'était un grand maximum. Or, quand Karajan l'enregistre, il en a huit, il s'en met donc déjà le double, et on en a plein les esgourdes ; en plus, après, du point de vue visuel, Clouzot - en accord avec Karajan - en a rajouté de façon à arriver à douze, alignées pour faire un effet de masse, se disant que s'il n'en mettait que trois ou quatre comme Beethoven, ou huit comme Karajan, ça ne serait pas assez spectaculaire. C'est un trucage visuel de Clouzot avec l'assentiment de Karajan : les contrebassistes jouaient de toute façon en play-back et vous auriez pu voir cinquante contrebasses que ça n'aurait rien changé au son, il n'aurait pas été plus fort. Il faut dire que Karajan a aussi mis au point un système de prise de son hollywoodien, qu'Emmanuel Krivine appelle une sorte de "sauce ». Karajan, c'est de la musique en sauce : il reste la viande, mais il y a beaucoup de choses autour. Il a énormément travaillé sur les prises de son très flatteuses et au moment où il a tourné les symphonies avec Clouzot, il a agi, du point de vue visuel, dans la même direction, dans la direction de l'énormité, du très grand confort.

Spectateur - Vous ne pensez pas que, sans les techniques modernes, le spectateur d'aujourd'hui risque d'être déçu?

58 J. D. - Je ne crois pas, ou, du moins, peut-être est-il un petit peu déçu au début, mais plus quand son écoute s'est adaptée. Tout à l'heure quelqu'un m'a parlé du son du clavecin qu'on a entendu hier soir au récital de Leonhardt; il disait qu'il était très faible. Mais c'est uniquement parce qu'on a l'habitude d'entendre du clavecin à la radio ou sur disque et qu'on a donc le même nombre de décibels que lorsqu'on écoute un orchestre symphonique. Avec un véritable instrument dans une vraie salle, on est un peu désorienté au début, et même un peu déçu, pourquoi pas, mais le temps passe et au bout de dix minutes l'oreille s'est adaptée et on entend très bien, exactement comme il faut. C'est la même chose quand on voit pour la première fois Chronique d'Anna Magdalena Bach : on a tellement l'habitude de voir à la télévision et au cinéma des films avec un plan toutes les secondes qu'on se dit : ça ne va quand même pas être comme ça pendant tout le film! Des plans fixes avec des chanteurs pendant des minutes et des minutes! Au début on est peut-être dérouté, mais on comprend très vite ce qui se passe dans ce film, ce qui est en jeu, et, une fois qu'on a compris, on écoute et on se rend compte qu'avant on croyait écouter, on ne faisait que croire qu'on écoutait, mais ce n'était pas vrai, on n'écoutait rien. Quand les caméras bougent tout le temps, vont partout, montent à toute vitesse, font des gros plans, des zooms, des surimpressions, des machins et des machins, on n'écoute plus. On a l'esprit complètement ailleurs, on regarde la moustache du chanteur, le pli de son pantalon, on regarde le cheveu qui n'est pas tout à fait au bon endroit, etc.

P. L. - On est hypnotisé par la forme trépidante, on est balloté et prisonnier. Il n'y a plus d'écoute véritable possible, de liberté. J. D. - La musique est parasitée.

61 François Narboni - Je suis d'accord avec ce que vous dites, Jacques, mais, dès qu'on filme, je me méfie de l'idéalisation du naturel en musique, qui veut qu'on doive 
exactement entendre l'instrument comme on l'entend dans une salle. Dès qu'il y a une caméra de télévision ou de cinéma, on passe dans un autre médium artistique qui vient se mélanger à la musique. Je n'ai pas vu le film de Clouzot mais qu'il y ait une contrebasse ou qu'il en ait quinze et qu'on en entende douze - ce dont sans doute Beethoven aurait rêvé - ça ne me gêne pas. La seule question est de savoir si ça me touche ou pas. Je me méfie d'un trop grand naturalisme, d'une trop grande objectivité : dans ce cas, pourquoi ne ferme-t-on pas les yeux pour ne rien voir? C'est comme pour le son direct : tout ce qu'on a entendu cet après-midi donne l'impression, peut donner l'impression, qu'il n'y a plus que le son direct, qu'il faut vénérer sa pureté, le sacraliser. Disons que pour les Straub ça marche, et vous avez tous parlé du pourquoi et du comment, expliqué les enjeux, les conséquences, mais c'est le son direct chez les Straub! C'est leur esthétique particulière! Car, dès le muet, l'histoire du cinéma s'est faite pour et avec un son qui n'était pas direct - les pianos qui accompagnaient les films muets n'étaient pas du son direct. La sacralisation du son direct, qui rejetterait le reste, me gêne, d'autant que je pourrais ajouter que le son direct c'est aussi le son de la télévision, c'est aussi le son des sitcoms, c'est aussi le son de plein d'affreux nanars et de films médiocres - ce n'est pas que les Straub. Cette sacralisation me gêne donc, comme l'idée de la pureté sonore : pourquoi un clavecin sur-amplifié, en quadriphonie, en cinq point un, ne serait pas bien aussi?

B. T. - Je crois que dans toutes ces questions il y a beaucoup d'enjeux qui sont assez différents et qui s'entremêlent. Certaines questions sont liées à l'histoire de l'écoute qu'est-ce-que c'est que d'écouter un instrument, qu'est-ce qu'entendre de la musique, peut-on faire abstraction de l'histoire, comment entendre aujourd'hui après John Cage un piano comme on l'entendait avant lui ? Et puis, il y a la question du son direct, sur laquelle je reviens. Ce que je voulais justement montrer en parlant notamment du choix des micros et de leur orientation, c'est que le son direct n'est absolument pas naturel. Il n'y a pas le son direct, il a des sons directs. Le son direct, c'est entièrement construit, c'est une construction comme le découpage, comme toute l'organisation et la composition d'un film, et, comme toujours, il s'agit de se donner les moyens de la construction dont on a besoin en envisageant tout ce que cela implique devant la caméra. Je voudrais terminer sur un point d'histoire de cinéma : il y a un moment où le son direct est arrivé au cinéma, c'est-à-dire le son synchrone, le fait de pouvoir sortir en extérieur - je ne parle pas des prises de vue en intérieur - avec une caméra et de prendre du son en même temps que l'image, et ce n'est pas du tout une chose qui date du parlant, c'est beaucoup plus postérieur, ça arrive à la charnière des années 1950-1960 et c'est un basculement. C'est un basculement - notamment permis par le Nagra, ce magnétophone à bande magnétique - car, pour la première fois, on voit et entend en même temps ce qui est filmé. C'est ce qui fait dire à Jean-Louis Comolli que le début du cinéma c'est 1959 parce que c'est le début du cinéma synchrone léger.

P. L. - Et tous les films de Danièle Huillet et Jean-Marie Straub, dont le premier date de 1962, sont issus de ce basculement.

64 J. D. - Et n'oublions pas quand même que Chronique d'Anna Magdalena Bach n'a pas que $\mathrm{du}$ son direct, puisqu'on a quelqu'un qui parle en voix off et que la voix off n'est pas du son direct... Ce qui veut dire que même chez Straub-Huillet, même chez eux, pour faire certaines choses on utilise cet artifice-là. Il s'agit de réserver telle chose à tel but et à telle fin et telle autre à tel autre; on sait tous que Straub a dit beaucoup de bien de 
Jacques Tati qui ne faisait pas spécialement du son direct, au contraire. Il y a une religion, mais il n'y a pas de dogmatisme, de sacralisation.

P. L. - Y-a-t-il d'autres questions, des commentaires tout simplement... J'avais cru entendre un début de question, pour Renato, je crois...

Bernard Muscat (Ciné Art) - Philippe, on me signale simplement qu'il ne faut pas que la conférence dure encore trop longtemps, parce que nous avons largement dépassé le temps qui était prévu, tu peux peut-être prendre une question et puis conclure....

P. L. - Ah bon ? Il y avait un temps de prévu ?... Mais nous avions encore beaucoup de choses à dire... Y a-t-il une dernière question alors?

B. M. - Si personne ne demande la parole, je m'adresserai à Renato Berta. Pouvez-vous nous dire, à partir d'un film comme Ouvriers, paysans, qui nous a beaucoup touchés, ou d'un autre, comment se passent, se passaient, les essais avec Jean-Marie Straub et Danièle Huillet? Quelle est votre part d'artisanat?

R. B. - Ça va être difficile de résumer ça en deux secondes. Il y a beaucoup de collègues opérateurs qui me disent que ça doit être chiant de tourner avec les Straub, ne serait-ce que parce que, pour eux, c'est tout le temps des plans fixes... Mais il y a une quantité de travail absolument gigantesque pour arriver déjà à cadrer, que ce soit un plan fixe ou pas. On pose une caméra, il faut décider de ce qu'on laisse hors du cadre. Et ça, avec Jean-Marie, même pour faire un plan fixe relativement simple, on y passe des heures. Il arrive évidemment que ça vienne tout de suite mais parfois c'est au bout de plusieurs heures : on cherche, on recule un peu la caméra, oui mais maintenant il y a une partie qui rentre, cet arbre-là, c'est pas bien, avance un peu, oui mais là c'est dommage parce qu'on perd autre chose, enfin bref, c'est un travail très pratique. Donc moi, je suis confronté au faire, je ne ressens pas les films après coup, une fois qu'ils sont finis, comme des critiques - vous voyez ce que je veux dire. Moi j'essaye, j'aide un metteur en scène à faire un film, avec les deux ou trois choses que je connais peut-être un peu mieux que lui ; j'essaye de contribuer, de nourrir le projet. J'ai tourné beaucoup de films en extérieur avec Danièle et Jean-Marie, et je dirais que le travail qu'on fait est, une fois qu'on a cadré, un travail d'observation, d'observation des comédiens confrontés à un texte pendant des heures. On tourne au minimum quatre prises bonnes ; alors, étant donnée la longueur des plans, vous voyez déjà ce que ça implique comme temps et comme travail, d'autant qu'un certain nombre de prises ne vont pas parce qu'il y a des erreurs, un tracteur qui passe au mauvais moment, un nuage un peu trop méchant qui a fait qu'on est parti trop en sous-exposition, etc. Parallèlement à ça, et c'est là où du coup ce travail est vraiment intéressant, on se laisse surprendre par tous les événements qui arrivent. Et si on peut se laisser surprendre - c'est ce que disait Philippe au début quand il parlait de ce qui surgit et qu'on accueille -, c'est grâce à tout ce qu'on a essayé de résoudre en amont en se donnant les moyens techniques nécessaires - le choix de la pellicule, du matériel... Il faut que j'ajoute que, contrairement à ce qui peut arriver avec un metteur en scène qu'on ne connaît pas et avec qui on doit commencer à comprendre ce qu'il entend par mettre en scène, ce qui est parfois acrobatique, avec Jean-Marie et Danièle on se connaissait depuis longtemps : la discussion est alors plus simple dans la mesure où il n'y a pas trop de psychologie, ou le peu qu'il y en a est vite mis de côté ; on peut se concentrer sur le côté pratique de la fabrication, et les mille problèmes qu'il y a toujours à résoudre. Enfin, je serais plus à l'aise pour répondre vraiment à votre question, et surtout ce serait, je crois, plus intéressant, plus concret, si un jour on avait l'occasion de prendre un film de Jean- 
Marie et Danièle, Ouvriers, paysans si vous voulez, et de le disséquer sur une table de montage, de mettre en évidence, à partir du plan de travail, les choix opérés, pourquoi on est parti dans une direction plutôt qu'une autre, ce serait pragmatique et simple.

B. M. - Merci et nous n'oublierons pas votre proposition passionnante.

71 P. L. - Merci à tous, et en particulier à Jacques Drillon, Renato Berta, Benoît Turquety et León García Jordán d'avoir participé à cette discussion. Merci également à toute l'équipe de Ciné Art. Bonne fin de journée.

\section{AUTEURS}

\section{PHILIPPE LAFOSSE}

Philippe Lafosse est l'auteur de L'Étrange cas de madame Huillet et de monsieur Straub. Il a assuré la rétrospective de l'œuvre des Straub à Nice, à la Villa Arson et au cinéma Reflet-Médicis à Paris en 2007. Responsable de publication de l'édition DVD des films des J.-M. Straub et D. Huillet aux éditions Montparnasse, il a également réalisé un film document sur les Straub : Dites-moi quelque chose.

\section{JACQUES DRILLON}

Jacques Drillon, docteur en linguistique, producteur à Radio France, fonde en 1978 Le Monde de la Musique, en 1995 la revue Symphonia et prend, en 1981, la succession de Maurice Fleuret à la rubrique « Musique classique » du Nouvel Observateur. Il est l'auteur de nombreuses publications sur la musique, le cinéma, le théâtre.

\section{RENATO BERTA}

Renato Berta, considéré comme l'un des plus grands directeurs de la photographie, travaille avec les plus exigeants des cinéastes : Daniel Schmid, Alain Tanner, Jean-Luc Godard, Patrice Chéreau, Alain Resnais, Manoel de Oliveira, Robert Guédiguian, Amos Gitaï et, depuis Othon (1969), avec Jean-Marie Straub et Danièle Huillet.

\section{BENOÎT TURQUETY}

Benoit Turquety est enseignant en cinéma à l'École nationale supérieure Louis Lumière et à l'Université Paris VIII/Vincennes-Saint-Denis. Il a soutenu en 2005 une thèse intitulée Huillet et Straub, «objectivistes » en cinéma; il est pour l'heure un des grands spécialistes actuels de leur œuvre.

\section{LÉON GARCIA JORDAN}

Léon Garcia-Jordan est né à Bogota en 1979. Photographe et réalisateur, il étudie d'abord la littérature et la philosophie, se passionne pour le cinéma avant de devenir photographe et de filmer les gestes d'artistes : peintres, musiciens, photographes. 